\title{
Bailing Out the Milky Way: Variation in the Properties of Massive Dwarfs Among Galaxy-sized Systems
}

\author{
Chris W. Purcell and Andrew R. Zentner
}

Department of Physics and Astronomy \& Pittsburgh Particle physics, Astrophysics and Cosmology Center (PITT PACC), University of Pittsburgh 15260 USA

E-mail: cpurcell@pitt.edu, zentner@pitt.edu

\begin{abstract}
Recent kinematical constraints on the internal densities of the Milky Way's dwarf satellites have revealed a discrepancy with the subhalo populations of simulated Galaxy-scale halos in the standard cold dark matter model of hierarchical structure formation. In particular, the Via Lactea II and Aquarius simulations both have large subhalos with internal densities that are larger than the constraints inferred for any Milky Way dwarf satellites. This has been dubbed the "too big to fail" problem, with reference to the improbability of large and invisible companions existing in the Galactic environment. In this paper, we argue that both the Milky Way observations and simulated subhalos are consistent with the predictions of the standard model for structure formation. Specifically, we show that there is significant variation in the properties of subhalos among distinct host halos of fixed mass and suggest that this can reasonably account for the deficit of dense satellites in the Milky Way. We exploit well-tested analytic techniques to predict the properties in a large sample of distinct host halos with a variety of masses spanning the range expected of the Galactic halo. Such techniques render the problem of estimating the variance in subhalo properties computationally feasible. The analytic model produces subhalo populations consistent with both Via Lactea II and Aquarius, and our results suggest that natural variation in subhalo properties suffices to explain the discrepancy between Milky Way satellite kinematics and these numerical simulations. At least $\sim 10 \%$ of Milky Way-sized halos host subhalo populations for which there is no "too big to fail" problem, even when the host halo mass is as large as $M_{\text {host }}=10^{12.2} h^{-1} \mathrm{M}_{\odot}$. Follow-up studies consisting of high-resolution simulations of a large number of Milky Way-sized hosts are necessary to confirm our predictions. In the absence of such efforts, the "too big to fail" problem does not appear to be a significant challenge to the standard model of cold dark matter halos undergoing hierarchical formation.
\end{abstract}

Keywords: Cosmology: theory — galaxies: formation — galaxies: evolution

ArXiv ePrint: 1208.4602 


\section{Contents}

1 Introduction 1

2 Methods 3

3 Reformulating the "Too Big To Fail" Problem 4

4 Results $\quad 6$

5 Discussion $\quad 10$

\section{Introduction}

In the standard paradigm describing structure growth in our universe, galaxies such as the Milky Way form within halos of dark matter $[1,2]$. This formation is hierarchical in the sense that small, self-bound clumps of dark matter collapse first into roughly virialized objects, subsequently merging at the nodes of sheets and filaments of dark matter to form ever larger halos. Most of the merging halos are disrupted within a few dynamical times and are no longer recognizable as distinct objects within the larger halo; however, some survive to the present day within the virialized regions of their host halos. The properties of these dark subhalos and their luminous components, such as the satellite galaxies of the Milky Way, are probes of the faintest substructure surrounding the Milky Way and may serve as sensitive testbeds of cosmological structure formation [3-8].

High-resolution simulations of $\Lambda \mathrm{CDM}$ cosmological evolution have been able to model the properties of subhalos within host halos similar to the Milky Way. However, the computational expense involved in such an undertaking has necessarily limited the number of experiments in which dwarf-sized satellites can be easily resolved at the ultra-faint scale $\left(L_{\text {dwarf }} \gtrsim 10^{5} \mathrm{~L}_{\odot}\right)$ to less than a dozen (taking as representative the set of Aquarius, GHALO, and Via Lactea II simulations in refs. [9] [10], and [11], respectively). Consequently, the diversity of subhalo characteristics and their natural variation among different host halos at fixed mass, as well as the details of those properties' dependence on halo mass, have only been subject to a relatively small number of studies.

Recent theoretical results in the context of Milky Way dwarf galaxy constraints suggest that the densest, most massive subhalos found in the Via Lactea II and Aquarius simulations have properties that are not commensurable with those of observed satellites in the Local Group [12]. This builds upon the early indications and possible cosmological implications of such a mismatch explored in ref. [4], and raises the question of whether the densest, most massive dwarf galaxies predicted by simulations are present in the real Milky Way environment. If these few largest halos are present, then why are they not luminous like their slightly less massive counterparts? In a properly cosmological context, it would seem that these putative satellites are "too big to fail", meaning that they are sufficiently more massive than the other Milky Way satellites that it is difficult to construct models in which these satellites remain invisible [12]. In such a scenario, these subhalos are either dense and somehow dark relics [12], or have had their baryonic content scoured via early feedback effects [13-15]. An alternative to these astrophysical solutions would conclude that these few 
most massive subhalos are not to be found and that this discrepancy is truly reflective of a critical failure in the $\Lambda \mathrm{CDM}$ structure formation paradigm (as proposed, for example, in the self-interacting CDM model of [16]).

In this work, we explore another possible solution to the "too big to fail" problem. We use analytic models to argue that the natural, statistical variation in subhalo densities from one host halo to another is significant and can affect the theoretical interpretation of satellite galaxy data. In particular, we suggest that the properties of the few densest, most massive Milky Way satellites do not pose a challenge to galaxy or structure formation theories. Rather, we argue that the observed satellite properties are consistent with being randomly drawn from among the statistical variety of subhalo populations that can be realized in Milky Way-sized halos, with a probability that is not negligible.

Halo-to-halo variation in subhalo properties is a natural consequence of the hierarchical structure-formation model as subhalos merge to form the contemporary host halo through an infinitude of distinct mass accretion histories. Any particular Galactic-scale halo is attended by a subhalo population with properties that are functions of this assembly history and likely a number of other variables. At present, we have some ability to constrain viable formation routes of the Milky Way. For example, our constraints on several properties of the thin and dynamically-cold Galactic disk imply that the host halo is unlikely to have suffered a minor merger (with mass ratio $\lesssim 10: 1$ ) passing through the disk within the past 5-8 Gyr [17, 18], before the ongoing Sagittarius impact and the imminent absorption of the Magellanic Clouds end that quiescent history at the present day [19-22]. However, existing constraints are broad and cannot be used to limit effectively the properties of the subhalos that should surround the Milky Way. Moreover, we do not have a comprehensive understanding of which variables are most important in determining the properties of the Milky Way subhalo population.

At the same time, we have only a poor handle on the Milky Way's total mass because stellar rotation curves only probe the central regions of the Milky Way halo and other probes yield a wide range of values [22-25]. Interestingly and unfortunately, this mass range is fraught with cosmologically-significant transitions that bear directly on galaxy formation [26-28], and numerical investigations have suggested that host halo mass is the largest determinant of subhalo properties in this particular context, with gas cooling and other baryonic effects taking a secondary role [29]. In addition, a recent statistical exploration of subhalo abundances in a simulated $\Lambda \mathrm{CDM}$ universe has illustrated the sensitivity of satellite populations to host halo mass, finding that Galaxy-sized halos with mass $M_{\text {host }} \lesssim 10^{12} \mathrm{M}_{\odot}$ are much more likely to have only three large companions (analogous to the Large and Small Magellanic Clouds and the disrupting Sagittarius dwarf) at the present-day [30].

We complement this and other numerical efforts with our ability to probe host-mass parameter space, and most importantly the intrinsic variation among subhalo properties at fixed host-mass, in much greater detail due to the computational advantages of our analytic approach. In the remainder of the paper, we itemize our methods and results. We describe the details of our formalism in $\S 2$, including a restatement in $\S 3$ of the "too big to fail" discrepancy in terms of a new variable describing central subhalo density. We give our basic results, culminating in probability distributions for this density-proxy variable, in $\S 4$, reserving $\S 5$ for discussion and interpretation in the context of future numerical experiments as well as achievable (and useful) observational constraints. 


\section{Methods}

Our investigations complement existing results in their focus on the quantification of the natural scatter in subhalo properties from one host halo to the next. In order to make such an estimate, it is necessary to derive the properties of subhalos in a very large number of host halos, a challenging task with direct numerical simulations because of the computational costs of such an effort. We overcome this difficulty by using an analytic technique to predict the properties of subhalo populations that approximates the results of numerical simulations at greatly reduced computational cost. The technique has been described in detail in [31], to which we refer the reader for further details, including a description of the algorithm as well as a demonstration that the model predictions for subhalo abundances, internal structures, and spatial distributions within host halos agree well with high-resolution numerical experiments in the regimes where the two techniques are commensurable. Several related techniques have been developed that produce results that are broadly similar but differ in some detail [e.g. 4, 32-38]. Similar methods have been applied to estimate the variance in putative dark matter annihilation signals yielded by galactic substructures [39].

The analytic calculations produce subhalo populations within individual, statistically distinct, host halos of a given mass. We refer to each host halo as a realization, because the subhalo properties within any given host are determined by a particular realization of the density field in the host environment. For each realization, the technique produces a catalog of subhalos, each being described by a variety of properties including: time at which the subhalo first merged into the host; subhalo mass at merger; final subhalo mass after evolving within the host to the present day; internal subhalo structure; and orbital position and velocity. These properties will enable us to make the estimates of interest to the present manuscript, since we can quantify the natural variation in subhalo properties among hosts by producing a very large number of distinct realizations at fixed host halo mass.

We work within a cosmological model described by the best-fitting parameters from the seven-year analysis of the Wilkinson Microwave Anisotropy Probe team [40]. We note that a halo's virial mass is calculated inside the region for which the mean density is greater than the mean matter density of the universe by a factor $\Delta$, that evolves in redshift from $\Delta(z=0) \sim 337$ to $\Delta(z) \sim 178$ for $z>1$, as in the fitting algorithm of ref. [41]. Throughout this work, we define subhalos as those self-bound objects inside this virial radius $R_{\text {vir }}$ at the present day. We examine three host halo masses that span an interesting range compared to both current very high-resolution numerical simulations, such as Via Lactea II and Aquarius, as well as current bounds on the halo mass of the Milky Way: $M_{\text {host }}=10^{11.8} h^{-1} \mathrm{M}_{\odot}$, $M_{\text {host }}=10^{12.0} h^{-1} \mathrm{M}_{\odot}$, and $M_{\text {host }}=10^{12.2} h^{-1} \mathrm{M}_{\odot}$. This mass range spans the various estimates for the present-day virial mass $M_{\text {host }}$ of the Milky Way, yielded by a number of methods including but not limited to the dynamical timing of Local Group galaxies and satellites of M31 [24, 42, $\left.M_{\text {host }} \simeq 10^{12.15} h^{-1} \mathrm{M}_{\odot}\right]$, as well as extrapolations drawn from the rotation curve of stars in the Galactic stellar halo $\left[23,10^{11.85 \pm 0.1} h^{-1} \mathrm{M}_{\odot}\right]$ and statistical comparisons with cosmological simulation results regarding the brightest Galactic satellites $\left[22,10^{11.92 \pm 0.2} h^{-1} \mathrm{M}_{\odot}\right]$. The Via Lactea II halo has a mass of $M_{\text {host }} \simeq 10^{12.2} h^{-1} \mathrm{M}_{\odot}$ [43, hereafter VL2]. In the Aquarius suite, six simulated halos span the mass range from $M_{\text {host }} \simeq 10^{12} h^{-1} \mathrm{M}_{\odot}$ to $M_{\text {host }} \simeq 10^{12.2} h^{-1} \mathrm{M}_{\odot}$ [9], and we note that Aquarius subhalos are identified within a radius defined by the virial overdensity $\Delta=250$ (and therefore a larger radius than all three models considered here, in which $R_{\mathrm{vir}} \sim 171,271,429 \mathrm{kpc}$ in order of ascending mass). Of these Aquarius subhalos, the analysis of ref. [12] adopts an outer limit 
of $R<300 \mathrm{kpc}$ for comparison to observed Galactic dwarfs.

At each of the three host halo masses that we model, we generate 10,000 realizations of halo substructure. These subhalo populations enable us to make an approximate assessment of the variation in satellite properties among different host halos at fixed host halo mass. Additionally, this large number of realizations enables us to sub-divide our samples while still making statistically-significant statements. For example, we explore cuts on subhalo accretion redshift and other properties in order to approximate some of the specific conditions that may pertain to the present state of the Milky Way disk, and the large size of our total realization set results in subsamples that are each statistically valuable in terms of Poisson error.

Before closing this section, we remind the reader that our technique is an approximation to direct simulations. The technique generally compares well to simulation results; however, as only a relatively small number of simulations exist in which substructure in Milky Waysized halos is very well resolved, it is not possible to rule out moderate systematic errors associated with this technique. Furthermore, this technique cannot be validated outside the range of scales that are well resolved by numerical simulations, and like the simulations to which we compare, it treats only the evolution of dark matter halos and neglects baryonic evolution. While the influence of baryons on subhalo structure on these scales is thought to be moderate at most, this remains a possible source of systematic error. These are important caveats, and they demand that the results of this study must be verified by future numerical simulation campaigns. Nevertheless, approximate techniques are presently the only way to estimate halo-to-halo variation in subhalo population properties at reasonable computational cost.

\section{Reformulating the "Too Big To Fail" Problem}

The "too big to fail" problem, as stated by [12], is based on the claim that the most massive subhalos in simulations are too dense to be consistent with existing constraints on the classical Galactic dwarf galaxies. Here, the internal densities of satellites are characterized in the space of $v_{\max }$ and $r_{\max }$, which are proxies for mass and size, respectively. As usual, we define $v_{\max }$ as the peak circular velocity in the satellite's rotation curve, which occurs at a radius $r_{\max }$ along that curve. These two parameters suffice to specify the subhalo density profile, provided it follows the typical Navarro-Frenk-White form [45]. In the standard picture of galaxy formation, more massive subhalos are expected to host brighter dwarf galaxies. The typical conclusion drawn in the literature thus far: if these massive and overly-dense satellites represent a generic prediction of $\Lambda \mathrm{CDM}$, then either something is badly wrong with our cosmological and astrophysical models, or there really are large and invisible dwarf galaxies around the Milky Way. The latter statement is at odds with the basic predictions of galaxy formation theory, since such massive systems would seem to be "too big to fail" in the baryonic sense.

Stellar kinematical data can yield a precise constraint on the subhalo masses of the Milky Way dwarf galaxies at particular radii [44]. These data constrain the structural parameters $v_{\max }$ and $r_{\max }$, and these constraints can be compared to the properties of subhalos in catalogs obtained from high-resolution numerical simulations of Galaxy-scale host halos (VL2 and Aquarius, as in ref. [12]), or our analytic host halo realizations. However, the data best constrain a degenerate combination of $v_{\max }$ and $r_{\max }$ [a point emphasized in 4]. The comparison of data with theoretical predictions is, therefore, a comparison along one 


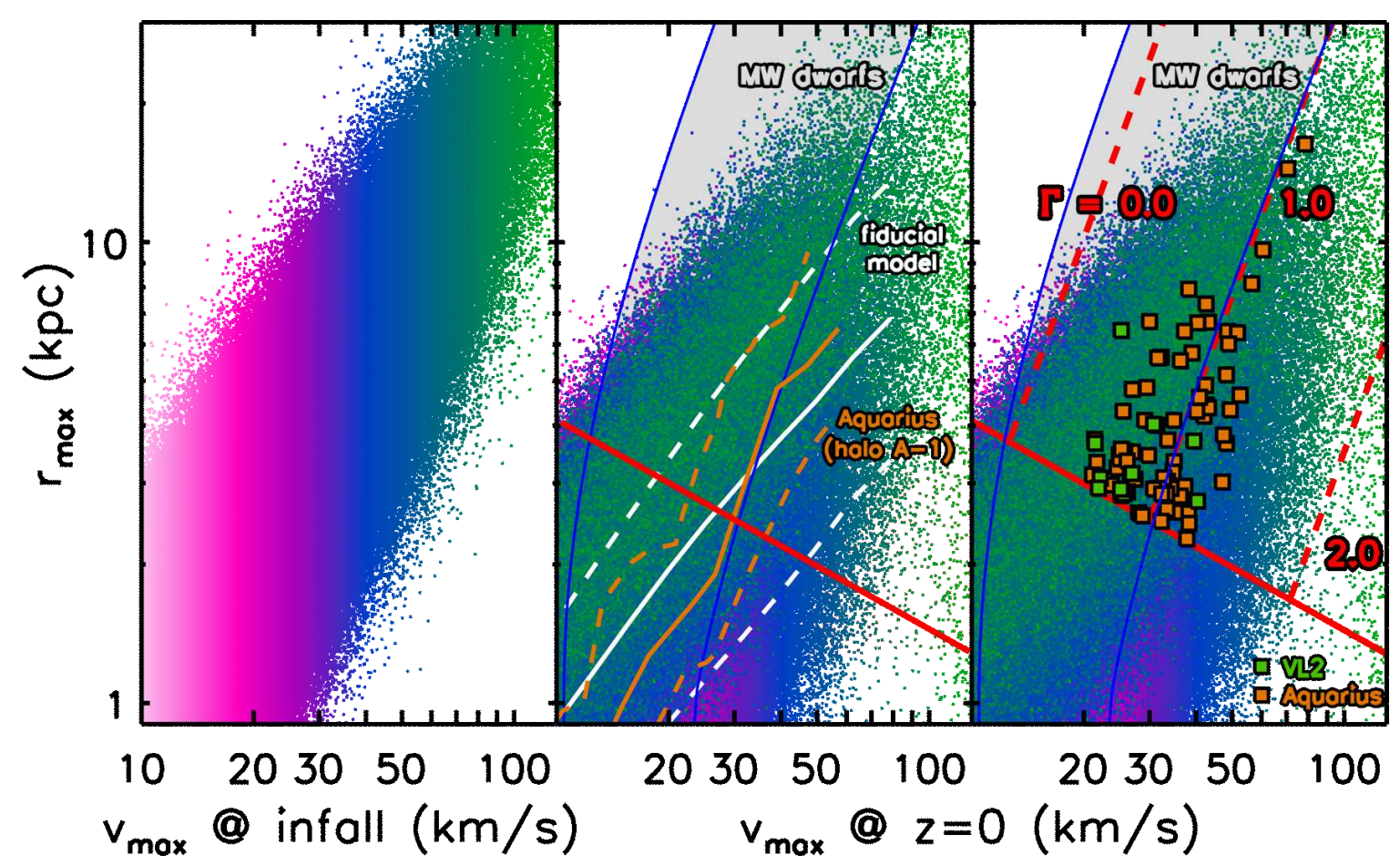

Figure 1. The distribution of all satellites in 10,000 realizations of a Galactic-scale host halo with total mass $M_{\text {host }}=10^{12.0} h^{-1} \mathrm{M}_{\odot}$, in the $v_{\max }-r_{\max }$ parameter space. As shown in the left panel, all subhalo points are colored by their value of $v_{\max }$ at infall through the virial radius of the host. In the center panel, we show in white solid and dashed lines the mean and 68\%-range of our fiducial $v_{\max }-r_{\max }$ distribution at redshift $z=0$ in comparison to the subhalo mean and $68 \%$-range for Aquarius halo A-1 (as published in [9]; shown in orange). Finally, in the right panel, we develop a parametrization fixed by the $2 \sigma$-constraint on the kinematic structures of the nine classical dwarfs around the Milky Way (denoted by the gray shaded region, as in [12]; see also [44]). The subhalo distributions in modern simulations of Milky Way-like host halos, including the VL2 simulation and all Aquarius subhalos in this regime, are shown in the right panel for satellites in the size regime where the observational envelope resembles a power-law, and we introduce the parameter $\Gamma$ describing the relevant direction of increasing density in the space of $\log \left(v_{\max }\right)-\log \left(r_{\max }\right)$.

particular dimension in the $\log \left(v_{\max }\right)-\log \left(r_{\max }\right)$ plane. A more detailed comparison may be possible, but the multidimensional parameter constraints on $v_{\max }$ and $r_{\max }$ for each dwarf are not available from ref. [12].

To make an approximate comparison of data with theory along the relevant dimension in the two-dimensional parameter space, we introduce a new parameter $\Gamma$, which represents the linear combination of $\log \left(v_{\max }\right)$ and $\log \left(r_{\max }\right)$ perpendicular to the $\operatorname{line}$ of $\log \left(r_{\max }\right)-\log \left(v_{\max }\right)$ degeneracy. Using the contours plotted in Figs. 1-2 of ref. [12], we define the parameter $\Gamma \equiv 1+\log \left(0.0014 v_{\max }^{2.2} / r_{\max }\right)$, which increases in a direction that is approximately orthogonal to the envelope of constraint on Milky Way dwarfs, simply expressing the formulation derived in ref. [44]. We normalize the scale of $\Gamma$ such that $\Gamma=1.0$ along the upper $2 \sigma$-bound of the allowable Milky Way dwarf region in the $v_{\max }-r_{\max }$ parameter space, and lower (higher) values of $\Gamma$ describe satellites that are less (more) dense and therefore less (more) discrepant with the few most massive Milky Way dwarfs. Roughly speaking, subhalos with $\Gamma>1$ are significantly denser than the observed Milky Way dwarfs. These are the satellites that are 
"too big to fail" according to the present literature [12].

In Figure 1, we illustrate the basic degeneracy of $v_{\max }$ and $r_{\max }$ and introduce our definition of $\Gamma$, by showing the $v_{\max }-r_{\max }$ space that contains the massive subhalos in all 10,000 realizations of the host halo of mass $M_{\text {host }}=10^{12.0} h^{-1} \mathrm{M}_{\odot}$ (our most similar model to the Aquarius A-1 halo for which the subhalo mean and scatter in $v_{\max }-r_{\max }$ have been published in [9]), at their infall into the virial radius of the host (in the leftmost panel) and the same population at the present day (in the center and right panels, where we develop the observationally-motivated constraint on Milky Way populations and the associated parameter $\Gamma$ defined above). We will compare predictions to data by evaluating distributions of the values of the parameter $\Gamma$ for subhalos with the Milky Way dwarf satellite data from [12]. As shown in Fig. 1, our subhalo populations generally agree with the Aquarius A-1 result in terms of mean and scatter, although in our region of interest involving relatively high-mass subhalos at the present-day, we sample many accretion events that are more rare by orders of magnitude than any surviving subhalos in Aquarius. These massive satellites are the rough counterparts to the large Galactic dwarfs such as the Sagittarius dwarf and the Magellanic Clouds, and we show that in general, these subhalos will evolve the most dramatically in

$v_{\max }-r_{\max }$ space, as their tidal mass-loss proceeds more efficiently than lower-mass satellites, which as a distribution remain relatively stationary.

The relevant direction indicated by $\Gamma$ is not a fundamental statement, because it involves observational data and will change as they evolve (or $\Gamma$ may cease to be useful altogether with significantly more powerful constraints). Moreover, a proper statistical accounting of the "too big to fail" problem would include the fact that each observed dwarf galaxy has a unique degeneracy direction in the $v_{\max }-r_{\max }$ plane by individually weighting each observational constraint and related error. We place such an effort beyond the scope of our present work, until future observations more clearly define these degeneracy directions as well as each individual dwarf's $\Gamma$-value; even with such data, only an extensive search of parameter space could identify individual realizations that are potentially analogous to the Milky Way in more detail. The basic goal of our analysis is to assess the relevance of the "too big to fail" problem as it is now typically constructed in the literature, i.e. the abundance of numerically-predicted subhalos outside the aggregated two-sigma envelope defined by observational constraints on Galactic dwarf satellites. We therefore adopt a parametrization that addresses this specific boundary, as a rough indicator of statistical consistency.

\section{Results}

Having defined the relevant dimension in the $v_{\max }-r_{\max }$ space in terms of the parameter $\Gamma$, which increases in the direction perpendicular to the upper- $2 \sigma$ boundary of the Milky Way subhalo densities, we consider the region shown in Figure 1 above which this bound closely resembles a power law. This corresponds roughly to satellites with $v_{\max } \gtrsim 30 \mathrm{~km} / \mathrm{s}$ at infall into the Galactic halo (or equivalently $r_{\max } \gtrsim 1-3 \mathrm{kpc}$ ); in general, subhalos evolve toward lower values of $v_{\max }$ as they lose mass due to tidal stripping after infall (and consequently also toward lower values of $r_{\max }$ ), and they do so approximately along the direction of degeneracy in kinematic solutions to the Milky Way satellites. As Fig. 1 demonstrates, the region of parameter space in which $0 \lesssim \Gamma \lesssim 1$ is amply populated by subhalos from our analytic models that would be consistent with the Galactic constraints. By comparison, massive subhalos in the VL2 and Aquarius simulations (appearing as shown in the analysis of [12]) are broadly 


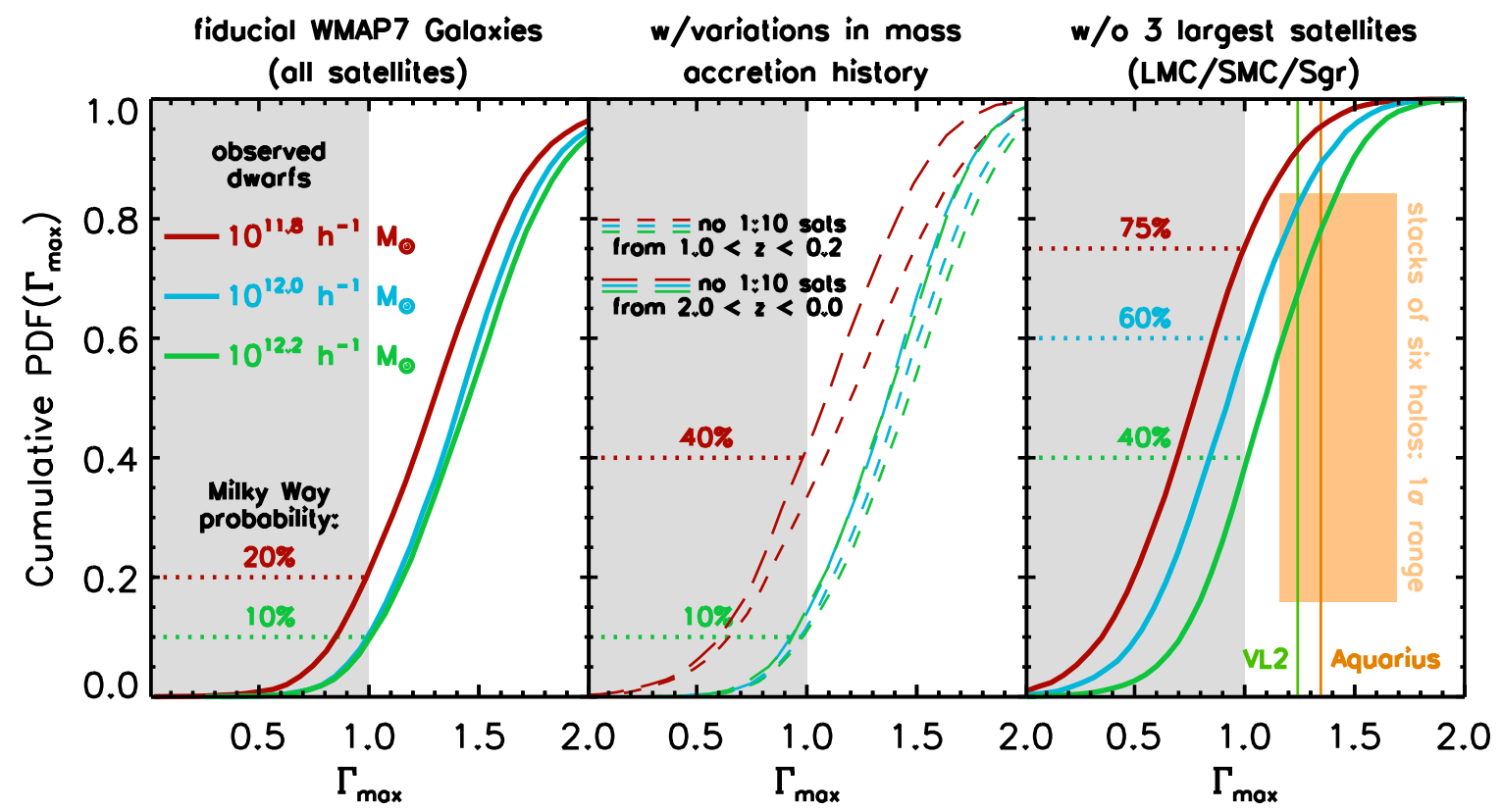

Figure 2. Cumulative probability-density functions, across all 10,000 realizations in each modeled host halo mass, describing the distribution of the largest subhalo-value per realization of the parameter $\Gamma_{\max }$, as described in $\S 4$. $\Gamma_{\max } \leq 1$ is our gross criterion for determining whether or not a particular subhalo population is consistent with the observed properties of the Milky Way dwarfs, hence the gray shaded region in all panels. In the left panel, we include all subhalos and evaluate the probability of drawing a dwarf galaxy population consistent with the observed Milky Way satellites. The center panel explores the effect of cutting the fiducial samples, requiring a relatively quiescent accretion history for inclusion (see relevant text in $\S 4$. Following the example of [12], in the right panel we remove from our fiducial samples the three densest subhalos, most similar to the presently-ongoing accretions of the Magellanic Clouds and the Sagittarius dwarf. The orange shaded region in this panel represents the mean $\pm 1 \sigma$ range of $\Gamma_{\max }=(1.37 \pm 0.21,1.51 \pm 0.18)$ for halo masses $M_{\text {host }}=\left(10^{12.0}, 10^{12.2}\right) h^{-1} \mathrm{M}_{\odot}$, where realizations are stacked in multiples of six for comparison to the Aquarius suite of halos also residing in this mass range.

consistent with the results of our analytic models, while also moderately populating the observationally-allowed region of the $v_{\max }-r_{\max }$ plane.

Any individual realization of a subhalo population is a particular sub-sample of this broad distribution of satellites. In order to make a simple comparison between the data and theoretical predictions, we characterize the subhalo population within a given host halo realization by a single parameter and explore the distribution of this parameter among host halo realizations. We select the maximum value of $\Gamma$ in any given realization as our summary statistic, $\Gamma_{\max }$. If $\Gamma_{\max } \leq 1$, then all subhalos in that realization would lie within the observationally-allowed region of the $v_{\max }-r_{\max }$ parameter space. In the absence of posterior distributions in $v_{\max }$ and $r_{\max }$ for each of the observed dwarf galaxies, it is difficult to make a more detailed comparison. We adopt $\Gamma_{\max } \leq 1$ as our gross criterion intended to describe satellite populations consistent with the Milky Way dwarf satellites as constrained by ref. [44] and adopted by ref. [12].

In Figure 2, we show the cumulative probability density for a random Milky Way realization to have a specific value of $\Gamma_{\max }$, allowing us to determine what fraction of host halos have a subhalo population where the maximally-dense satellite has $\Gamma_{\max } \leq 1.0$. Including 
all satellites and all realizations, the probability of meeting this constraint is $\sim 10-20 \%$, increasing as modeled host mass decreases. This already suggests that the "too big to fail" discrepancy is not a severe problem, even if stated in its most constraining form. Rather, the lack of observed dwarf satellites with "high densities" (high values of $\Gamma_{\max }$ ) may well be accommodated within the standard model of cosmological structure formation, given the variation in subhalo populations from one host halo to the next.

Beyond this comparison, it is interesting to explore the influence of particular cuts on halo formation on the distribution of $\Gamma_{\max }$ and how any such cuts may influence our interpretation of the evolution of the Milky Way in light of the "too big to fail" conjecture. We are motivated by observational clues that the Galactic assembly history has been relatively quiescent over the past several Gyr [17], and by theoretical work that has shown the presentday Milky Way to be incompatible with any significant mass accretion events during that time [18]. If we thus impose any reasonable kind of relatively quiescent mass accretion history, as shown in the two such cuts displayed in the center panel of Fig. 2, the probability of randomly drawing a Galactic satellite population increases to $\sim 10-40 \%$ for our range of host masses. Specifically, we identify the accretion-event scale of interest by the mass ratio $M_{\text {host }}: M_{\text {sat }} \lesssim 10: 1$, in accordance with the rough constraints derived from the thin and dynamically-cold state of the Milky Way disk. The most stringent cut we impose requires that no such event has occurred since $z=2$, while the less severe cut eliminates $10: 1$ events since $z=1$ while also allowing for recent minor mergers after $z=0.2$. In all halo-mass cases, increasing the quiescence of the assembly history only fractionally increases the probability (according to the $\Gamma_{\max }$ analysis) that a Galaxy-analog realization will be consistent with the Milky Way satellite constraints.

Convolving this analysis with the fact that massive minor mergers are relatively rare in Galaxy-scale halos at the present-day, although they appear to be occurring in triplicate for the Milky Way today (in the forms of the disrupting Sagittarius dwarf and infalling Magellanic Clouds), we have a sense now not only of the discriminative power in $\Gamma_{\max }$, but also of the subtlety involved in characterizing halo formation histories by the properties of their most massive subhalos. To avoid contaminating the sample of classical dwarf satellites with these three ongoing accretion events in the Milky Way, the analyses of [12] and related investigations removed all subhalos in the VL2 and Aquarius catalogs with $v_{\max }>80 \mathrm{~km} / \mathrm{s}$ at the present day. With a large statistical sample of realizations, we can specifically remove the three most massive satellites from each realization, thus masking on average the three most recent Galactic accretion events by not allowing them to set $\Gamma_{\max }$ in any host halo. The Magellanic Clouds and Sagittarius are not included in the [12] analysis; however, it is not entirely obvious which subhalos to remove from a theoretically-constructed subhalo catalog to account for this effect. Removing the three most massive subhalos is the most optimistic strategy in terms of driving predictions to agree with data, and we adopt this test in light of the above demonstration that the "too big to fail" problem is a concordancecosmology result for at least $10 \%$ of Galaxy-sized halos without culling any satellites from the catalogs at all. Certainly a more sophisticated method for describing Sagittarius and the Magellanic Clouds would be desirable, as would tighter constraints on individual $\Gamma$-values for the observed classical dwarfs, but considerable uncertainty would still remain regarding the properties of their attendant dark matter halos, and the intrinsic scatter in halo/stellar-mass relations further complicates the issue [47, 48].

After trimming each subhalo catalog in this way and recalculating the cumulative probability density, we show in the right panel of Fig. 2 that the observed Milky Way satellite 


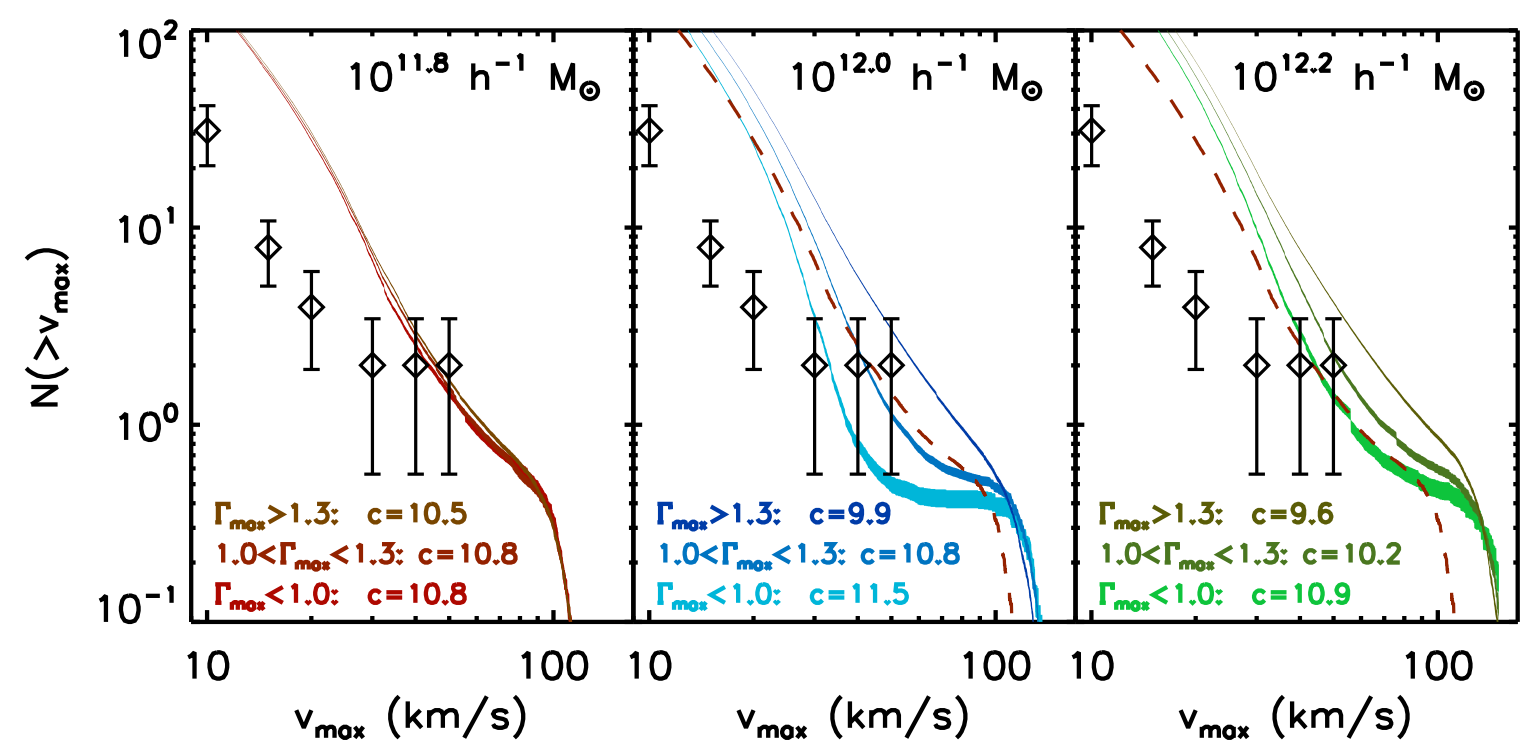

Figure 3. The cumulative velocity function $N\left(>v_{\max }\right)$ describing the number of subhalos with circular velocity values larger than $v_{\max }$, as in typical "missing satellite" analyses; all solid curves represent the analytic results, where the thickness of the line represents twice the Poisson error. In each halo-mass model, we divide the catalog into samples binned by $\Gamma_{\max }$, finding for the two largest host masses that realizations with lower values of the density-proxy parameter have fewer overall satellites, especially at the more massive end of the subhalo spectrum (although even in this regime of $\Gamma_{\max }$, there are rare halos hosting very large satellites from recent accretion events). Note that decreasing $\Gamma_{\max }$ correlates with an increase in the mean value of the host halo's concentration parameter $c$, which in all cases has a Poisson error on the mean of less than 0.1). In the medium- and high-mass panels, we show the mean low-mass behavior (dashed red) to illustrate the possible degeneracy between halo mass and slope of the cumulative velocity function of satellites. For comparison, we overplot the Milky Way satellite velocity function with Poisson error-bars as presented by [46], noting that the low-mass data points likely underestimate the true Galactic dwarf distribution by a factor of $\sim 5$ or more, due to luminosity-bias and sky-coverage issues as elaborated by [5].

population becomes much more likely in the analytic distribution, occurring in $\sim 40-75 \%$ of the realizations and increasing with decreasing host mass as above. For comparison, our adopted samples from the VL2 and Aquarius simulations have $\Gamma_{\max } \simeq 1.24$ and 1.34, respectively (note that the Aquarius value represents the most dense satellite in a suite of six host halos with virial masses spanning the range between our two most massive models, and also that the VL2 host halo is most similar to our heaviest modeled virial mass). Stacking our catalogs in multiples of six realizations for a more direct comparison to Aquarius, we find the mean $\pm 1 \sigma$ range of $\Gamma_{\max }=(1.23 \pm 0.21,1.37 \pm 0.21,1.51 \pm 0.18)$ for halo masses $M_{\text {host }}=\left(10^{11.8}, 10^{12.0}, 10^{12.2}\right) h^{-1} \mathrm{M}_{\odot}$ respectively. We also show in Fig. 2 these mediumand high-mass ranges of $\Gamma_{\max }$ in stacks of six realizations, since this mass regime is more properly compared to the Aquarius host halos. Based on this analysis, the values of $\Gamma$ in our subhalo populations seem to be broadly consistent with the results of the VL2 and Aquarius simulations, suggesting at least that we are not subject to an egregious systematic error.

A relatively low value of $\Gamma_{\max }$ represents a dearth of dense, massive subhalos in a particular host halo, so it is instructive to consider the subhalo populations within low- $\Gamma_{\max }$ hosts. This is interesting for several reasons. First, it may lead toward novel predictions that can be used to test our solution, based on large variation in subhalo populations among 
host halos, of the "too big to fail" problem. Second, it may have some bearing on the longstanding "missing satellite problem" [49, 50]. In Figure 3, we show the cumulative velocity functions $N\left(>v_{\max }\right)$ describing the distribution of satellites in each of our three models, subdividing each host halo mass into three samples binned by the value of $\Gamma_{\max }$ of each realization. In the two largest host-mass models, the subsets with progressively smaller $\Gamma_{\max }$ have steeper $N\left(>v_{\max }\right)$ functions with lower overall normalizations, which also correspond to samples with progressively larger host halo concentration values. The variation between subsets is much less pronounced in the low-mass $M_{\text {host }}=10^{11.8} h^{-1} \mathrm{M}_{\odot}$ model, in which the host concentration values are similar across subsets of $\Gamma_{\max }$. For comparison, we show the velocity function of Milky Way dwarfs (including those satellites typically called "ultrafaint") as obtained by ref. [46], noting that the low-mass end of this data set is subject to the sky-coverage and luminosity-bias issues elaborated by ref. [5] among others.

\section{Discussion}

Using stellar kinematical data, ref. [12] compared constraints on the structural properties of the halos that host the dwarf satellite galaxies of the Milky Way with the structural parameters predicted by a number of numerical simulations of the formation of Milky Waysized dark matter halos. In doing so, they identified a discrepancy: too many numerical subhalos have $v_{\max }$ values larger than would be expected for systems of their size, when set against these Galactic observations. If $\Lambda \mathrm{CDM}$ cosmological predictions and/or astrophysical models are not too badly wrong, then do massive invisible dwarfs exist around the Milky Way? How could such putative objects have failed to light up with star formation? The authors of ref. [12] dubbed this issue the "too big to fail" problem.

Soon afterward, ref. [30] showed that one way to mitigate the "too big to fail" discrepancy is for the Milky Way to reside within a host dark matter halo at the lower-mass range of contemporary constraints on the size of the Milky Way halo. Specifically, this investigation of the Millennium Simulation statistics found that for a threshold $v_{\max }=30 \mathrm{~km} / \mathrm{s}$, fully $\sim 40 \%$ of halos with host mass $M_{\text {host }}=10^{12} M_{\odot}=10^{11.85} h^{-1} \mathrm{M}_{\odot}$ host three or fewer satellites larger than the threshold (compared to the eight such satellites typical of the Aquarius halos). For halos more massive than $M_{\text {host }} \gtrsim 2 \times 10^{12} M_{\odot}=10^{12.15} h^{-1} \mathrm{M}_{\odot}$, this probability drops below $5 \%$ and quickly vanishes thereafter with increasing halo mass. Our results are certainly in broad agreement with this conclusion, although the two predictions do not probe identical probability distributions; the summary statistic $\Gamma$ involves $r_{\max }$ as well as the $v_{\max }$ distribution probed by ref. [30]. We therefore focus on the parameter $\Gamma$ because it can directly address the degeneracies in halo properties permitted by the data, while noting that a simple translation of our $\Gamma_{\max }<1$ criterion into $v_{\max }<30 \mathrm{~km} / \mathrm{s}$ results in $\sim 37 \%$ of $M_{\text {host }}=10^{11.8} \mathrm{M}_{\odot}$ hosts satisfying the constraints, in significant agreement with the sample analyzed by ref. [30].

Proposed solutions to the "too big to fail" problem, in looking for a means to reduce subhalo internal density, have ranged from the cosmological (if satellite centers are scoured by self-interacting dark matter as in ref. [16]) to the astrophysical (if dwarf galaxies sweep out dark mass via feedback effects as in refs. [13-15]). In this paper, we explore an additional way in which the "too big to fail" discrepancy may be mitigated. In particular, we propose that the large variation in subhalo populations among different host halos can explain the dearth of large, dense subhalos orbiting the Milky Way without any making any adjustments to the host halo mass or accounting for baryonic feedback processes. For a host halos at 
the high-mass end of the generally accepted range for the host halo of the Milky Way, $M \approx 10^{12.2} h^{-1} \mathrm{M}_{\odot}$, we find that at least $\sim 10 \%$ of all host halos would harbor a subhalo population consistent with the observed stellar kinematics of the Milky Way dwarfs. This probability can be considerably higher if the three objects neglected in the [12] analysis (Sagittarius and the Magellanic Clouds) exhibit any of the highest densities among the Milky Way subhalo population.

In formulating our comparison of theoretical predictions with observational constraints, we have introduced a new density-proxy parameter $\Gamma$, that roughly runs perpendicular to the degeneracy between the subhalo structural parameters $v_{\max }$ and $r_{\max }$ exhibited by the data. Our detailed results are statements about the approximate probability distribution of the parameter $\Gamma$ among subhalos in different hosts. Our study has not identified additional diagnostics that can be used to characterize the subset of host halo merger histories that produce satellite populations not subject to the "too big to fail" problem. From Fig. 2, we see that enforcing a recent epoch of relative quiescence on the host halo does not significantly increase probability for harboring an acceptable subhalo population. The one exception to this conclusion is for the lowest host halo mass, $M_{\text {host }}=10^{11.8} h^{-1} \mathrm{M}_{\odot}$. The probability increase reflects the fact that less massive halos are more likely to have experienced minor mergers of a given mass ratio by the present day [51], and this relative homogeneity among host halos also manifests in the cumulative velocity functions and average concentration parameters $c$ shown in Fig. 3, which do not vary outside the Poisson error on the mean for the $M_{\text {host }}=10^{11.8} h^{-1} \mathrm{M}_{\odot}$ model.

Using the large variance of available merger histories to test the observational consistency of any particular realization's subhalo population could be an avenue of potential interest in an era of near-field cosmology that seeks to connect specific substructure in the Milky Way to characteristics of Galaxy-formation theory. Our rough test along those lines in the present work differentiates mainly between systems that have undergone their allotted minor mergers versus those that have not yet been impacted by high-mass subhalos; in a sense, we already know that the Galaxy is part of the latter class, due to the unusually-thin and dynamically-cold state of the stellar disk, and that in fact it is presently undergoing multiple minor mergers. However, to get a much more detailed handle on the assembly history of the Milky Way by inspecting analytic distributions of $\Gamma$ and $\Gamma_{\max }$, one would require a reasonable estimate of $v_{\max }$ for each individual satellite galaxy's host halo, and it is unclear what (if any) fundamental science could be done with such an effort, beyond refining the correspondence between numerical results, theoretical predictions, and observational constraints (which we argue here to be statistically sound, in contrast to the claims implicit to the "too big to fail" conjecture).

Fig. 3 shows subtle distinctions between the properties of host systems at the two larger masses $\left(M_{\text {host }}=10^{12.0} h^{-1} \mathrm{M}_{\odot}\right.$ and $\left.M_{\text {host }}=10^{12.2} h^{-1} \mathrm{M}_{\odot}\right)$ that pass the "too big to fail" test and those that do not. Hosts halos of these masses that contain subhalo populations that are grossly consistent with the Milky Way satellite populations also generally have concentrations that are slightly higher than average, though this systematic offset is smaller than the dispersion in concentration at fixed mass [52-54]. This weak trend is broadly consistent with the sense that the Milky Way may be an unusually small disk galaxy among similarly-sized systems, in terms of stellar mass as well as angular momentum [17], as $\Lambda$ CDM models predict that disk size increases with decreasing host halo concentration [52]. This implication also appears to be consistent with recent measurements that correlate satellite concentration with host stellar mass and total halo mass, in nearby disk-dominated galaxies 
[55].

A possibly more dramatic feature of subhalo populations within host halos in our highermass samples that are not in conflict with Milky Way data is that they exhibit relatively steeper cumulative velocity functions, as shown in Fig. 3. At best, this only slightly ameliorates the discrepancy that has come to be known as the "missing satellite" problem, if we take low $\Gamma_{\max }$ samples to represent possible Milky Way realizations. However, as large-scale surveys of the Galactic environment complete the local census of dwarf satellites, the faintend slope may probe this prediction of models that solve the "too big to fail" problem by exploiting the large variance in subhalo populations. We note, however, that Fig. 3 appears to indicate that halo mass and velocity-function slope may be somewhat degenerate, and therefore a full exploration of this parameter space by observations of substructure in extragalactic systems could be useful in discriminating between the competing effects we discern here.

Interestingly, the steeply-sloped realizations with low $\Gamma_{\max }$ also display a plateau beneath a value of unity in the subhalo velocity function. This reflects the fact that some relatively rare Galaxy-sized halos may have a very massive companion with $v_{\max }>60 \mathrm{~km} / \mathrm{s}$, with such a subhalo having necessarily fallen in recently (since dynamical friction destroys massive objects quickly). We note that the Milky Way dwarf population mimics this behavior somewhat, within observational Poisson error of the analytic plateau, due to the presence of three large satellites with $v_{\max }>60 \mathrm{~km} / \mathrm{s}$ as well as the absence of any moderately-massive subhalos between $30<v_{\max }<60 \mathrm{~km} / \mathrm{s}$, supporting the conclusion that the low $\Gamma_{\max }$ description of the Galactic dwarfs is consistent with a flat velocity function in this intermediate range, and favors the presence of one or possibly more large systems with relatively higher $v_{\max }$. In the particular case of the Milky Way, this plateau occurs at three large satellites, due to the conjoined Magellanic Clouds and the currently-disrupting Sagittarius dwarf galaxy.

In summation, we have used analytic models of subhalo populations to argue that the absence of luminous Milky Way satellite galaxies residing in halos of high density may not indicate a problem with contemporary theories of structure formation or galaxy formation. Rather, we have argued that the non-negligible variation in subhalo populations may reasonably account for this deficit. We estimate that at least $10 \%$ of halos could be consistent with observations without any requirement that the host halo of the Milky Way have a mass toward the lower range of contemporary constraints. There are several implications of this result. First, it suggests that there is no need to consider relatively low-mass hosts for the Milky Way (though such may certainly be the case, and could be a large part of the solution). Second, it shows that the "too big to fail" problem cannot be a statement of high statistical significance. Further study is necessary to confirm or refute our argument. In particular, we have, by necessity, used approximate techniques to predict the properties of dark matter subhalos so a large numerical simulation campaign will be necessary to test our predictions. In performing such a follow-up study, it must be borne in mind that selecting particular halos for high-resolution resimulation using accretion history or halo structural information $[9,11]$ can introduce biases; however, we have not been able to identify clear biases in our analytic models. Furthermore, we have neglected the effects of baryons and these may need to be considered more carefully. Finally, once a large set of simulated subhalo populations is available, it will be imperative to conduct a full statistical comparison of theoretical predictions with stellar kinematical data. Such follow-up studies may, indeed refute our argument perhaps by identifying undiagnosed systematic errors in our methods. However, absent such detailed follow-up, the "too big to fail" problem is unlikely to pose a serious challenge to 
the standard, hierarchical cold dark matter model of structure growth; rather than being a potential wound to the predictive power of $\Lambda \mathrm{CDM}$, it may instead represent an exhibition of its productive variety.

\section{Acknowledgments}

We would like to thank Michael Boylan-Kolchin, Andrew Hearin, and Mike Kuhlen for useful discussions. This work was funded by the University of Pittsburgh, by the Pittsburgh Particle physics, Astrophysics, and Cosmology Center (PITT PACC), and by the National Science Foundation through Grants NSF PHY 0968888 and NSF AST 1108802.

\section{References}

[1] S. D. M. White and M. J. Rees, Core condensation in heavy halos - A two-stage theory for galaxy formation and clustering, Monthly Not. Royal Ast. Soc. 183 (May, 1978) 341-358.

[2] G. R. Blumenthal, S. M. Faber, J. R. Primack, and M. J. Rees, Formation of galaxies and large-scale structure with cold dark matter, Nature 311 (Oct., 1984) 517-525.

[3] M. Kamionkowski and A. R. Liddle, The Dearth of Halo Dwarf Galaxies: Is There Power on Short Scales?, Physical Review Letters 84 (May, 2000) 4525-4528, [astro-ph/].

[4] A. R. Zentner and J. S. Bullock, Halo Substructure and the Power Spectrum, Astrophys. J. 598 (Nov., 2003) 49-72, [astro-ph/].

[5] E. J. Tollerud, J. S. Bullock, L. E. Strigari, and B. Willman, Hundreds of Milky Way Satellites? Luminosity Bias in the Satellite Luminosity Function, Astrophys. J. 688 (Nov., 2008) 277-289, [arXiv:0806.4381].

[6] J. S. Bullock, K. R. Stewart, M. Kaplinghat, E. J. Tollerud, and J. Wolf, Stealth Galaxies in the Halo of the Milky Way, Astrophys. J. 717 (July, 2010) 1043-1053, [arXiv:0912.1873].

[7] A. S. Font, A. J. Benson, R. G. Bower, C. S. Frenk, A. Cooper, G. De Lucia, J. C. Helly, A. Helmi, Y.-S. Li, I. G. McCarthy, J. F. Navarro, V. Springel, E. Starkenburg, J. Wang, and S. D. M. White, The population of Milky Way satellites in the $\Lambda$ cold dark matter cosmology, Monthly Not. Royal Ast. Soc. 417 (Oct., 2011) 1260-1279, [arXiv:1103.0024].

[8] V. Rashkov, P. Madau, M. Kuhlen, and J. Diemand, On the Assembly of the Milky Way Dwarf Satellites and Their Common Mass Scale, Astrophys. J. 745 (Feb., 2012) 142, [arXiv: 1106.5583].

[9] V. Springel, J. Wang, M. Vogelsberger, A. Ludlow, A. Jenkins, A. Helmi, J. F. Navarro, C. S. Frenk, and S. D. M. White, The Aquarius Project: the subhaloes of galactic haloes, Monthly Not. Royal Ast. Soc. 391 (Dec., 2008) 1685-1711, [arXiv:0809.0898].

[10] J. Stadel, D. Potter, B. Moore, J. Diemand, P. Madau, M. Zemp, M. Kuhlen, and V. Quilis, Quantifying the heart of darkness with GHALO - a multibillion particle simulation of a galactic halo, Monthly Not. Royal Ast. Soc. 398 (Sept., 2009) L21-L25, [arXiv:0808. 2981].

[11] J. Diemand, M. Kuhlen, and P. Madau, Formation and Evolution of Galaxy Dark Matter Halos and Their Substructure, Astrophys. J. 667 (Oct., 2007) 859-877, [astro-ph/].

[12] M. Boylan-Kolchin, J. S. Bullock, and M. Kaplinghat, Too big to fail? The puzzling darkness of massive Milky Way subhaloes, Monthly Not. Royal Ast. Soc. 415 (July, 2011) L40-L44, [arXiv: 1103.0007].

[13] A. di Cintio, A. Knebe, N. I. Libeskind, G. Yepes, S. Gottlöber, and Y. Hoffman, Too small to succeed? Lighting up massive dark matter subhaloes of the Milky Way, Monthly Not. Royal Ast. Soc. 417 (Oct., 2011) L74-L78, [arXiv:1107.5045]. 
[14] M. Boylan-Kolchin, J. S. Bullock, and M. Kaplinghat, The Milky Way's bright satellites as an apparent failure of $\Lambda$ CDM, Monthly Not. Royal Ast. Soc. 422 (May, 2012) 1203-1218, [arXiv: 1111.2048].

[15] A. Zolotov, A. M. Brooks, B. Willman, F. Governato, A. Pontzen, C. Christensen, A. Dekel, T. Quinn, S. Shen, and J. Wadsley, Baryons Matter: Why Luminous Satellite Galaxies Have Reduced Central Masses, ArXiv e-prints (June, 2012) [arXiv:1207.0007].

[16] M. Vogelsberger, J. Zavala, and A. Loeb, Subhaloes in self-interacting galactic dark matter haloes, Monthly Not. Royal Ast. Soc. (May, 2012) 3127, [arXiv:1201.5892].

[17] F. Hammer, M. Puech, L. Chemin, H. Flores, and M. D. Lehnert, The Milky Way, an Exceptionally Quiet Galaxy: Implications for the Formation of Spiral Galaxies, Astrophys. J. 662 (June, 2007) 322-334, [astro-ph/].

[18] C. W. Purcell, S. Kazantzidis, and J. S. Bullock, The Destruction of Thin Stellar Disks Via Cosmologically Common Satellite Accretion Events, Astrophys. J. Lett. 694 (Apr., 2009) L98-L102, [arXiv:0810.2785].

[19] D. R. Law and S. R. Majewski, The Sagittarius Dwarf Galaxy: A Model for Evolution in a Triaxial Milky Way Halo, Astrophys. J. 714 (May, 2010) 229-254, [arXiv:1003.1132].

[20] C. W. Purcell, J. S. Bullock, E. J. Tollerud, M. Rocha, and S. Chakrabarti, The Sagittarius impact as an architect of spirality and outer rings in the Milky Way, Nature 477 (Sept., 2011) 301-303, [arXiv:1109.2918].

[21] G. Besla, N. Kallivayalil, L. Hernquist, B. Robertson, T. J. Cox, R. P. van der Marel, and C. Alcock, Are the Magellanic Clouds on Their First Passage about the Milky Way?, Astrophys. J. 668 (Oct., 2007) 949-967, [astro-ph/].

[22] M. T. Busha, P. J. Marshall, R. H. Wechsler, A. Klypin, and J. Primack, The Mass Distribution and Assembly of the Milky Way from the Properties of the Magellanic Clouds, Astrophys. J. 743 (Dec., 2011) 40, [arXiv:1011.2203].

[23] X. X. Xue, H. W. Rix, G. Zhao, P. Re Fiorentin, T. Naab, M. Steinmetz, F. C. van den Bosch, T. C. Beers, Y. S. Lee, E. F. Bell, C. Rockosi, B. Yanny, H. Newberg, R. Wilhelm, X. Kang, M. C. Smith, and D. P. Schneider, The Milky Way's Circular Velocity Curve to $60 \mathrm{kpc}$ and an Estimate of the Dark Matter Halo Mass from the Kinematics of ²400 SDSS Blue Horizontal-Branch Stars, Astrophys. J. 684 (Sept., 2008) 1143-1158, [arXiv:0801.1232].

[24] Y.-S. Li and S. D. M. White, Masses for the Local Group and the Milky Way, Monthly Not. Royal Ast. Soc. 384 (Mar., 2008) 1459-1468, [arXiv:0710.3740].

[25] N. Przybilla, A. Tillich, U. Heber, and R.-D. Scholz, Weighing the Galactic Dark Matter Halo: A Lower Mass Limit From the Fastest Halo Star Known, Astrophys. J. 718 (July, 2010) 37-42, [arXiv: 1005.5026].

[26] S. Codis, C. Pichon, J. Devriendt, A. Slyz, D. Pogosyan, Y. Dubois, and T. Sousbie, Connecting the cosmic web to the spin of dark halos: implications for galaxy formation, ArXiv e-prints (Jan., 2012) [arXiv:1201.5794].

[27] C. Pichon, D. Pogosyan, T. Kimm, A. Slyz, J. Devriendt, and Y. Dubois, Rigging dark haloes: why is hierarchical galaxy formation consistent with the inside-out build-up of thin discs?, Monthly Not. Royal Ast. Soc. 418 (Dec., 2011) 2493-2507, [arXiv:1105.0210].

[28] Q. Guo, S. White, C. Li, and M. Boylan-Kolchin, How do galaxies populate dark matter haloes?, Monthly Not. Royal Ast. Soc. 404 (May, 2010) 1111-1120, [arXiv:0909.4305].

[29] S. Geen, A. Slyz, and J. Devriendt, Satellite Survival in Highly Resolved Milky Way Class Halos, ArXiv e-prints (Apr., 2012) [arXiv:1204.3327].

[30] J. Wang, C. S. Frenk, J. F. Navarro, L. Gao, and T. Sawala, The missing massive satellites of 
the Milky Way, Monthly Not. Royal Ast. Soc. (July, 2012) 3369, [arXiv:1203.4097].

[31] A. R. Zentner, A. A. Berlind, J. S. Bullock, A. V. Kravtsov, and R. H. Wechsler, The Physics of Galaxy Clustering. I. A Model for Subhalo Populations, Astrophys. J. 624 (May, 2005) 505-525, [astro-ph/].

[32] A. R. Zentner, The Excursion Set Theory of Halo Mass Functions, Halo Clustering, and Halo Growth, International Journal of Modern Physics D 16 (2007) 763-815, [astro-ph/].

[33] J. Gan, X. Kang, F. C. van den Bosch, and J. Hou, An improved model for the dynamical evolution of dark matter subhaloes, Monthly Not. Royal Ast. Soc. 408 (Nov., 2010) 2201-2212, [arXiv: 1007.0023].

[34] X. Yang, H. J. Mo, Y. Zhang, and F. C. van den Bosch, An Analytical Model for the Accretion of Dark Matter Subhalos, Astrophys. J. 741 (Nov., 2011) 13, [arXiv:1104.1757].

[35] J. E. Taylor and A. Babul, The evolution of substructure in galaxy, group and cluster haloes I. Basic dynamics, Monthly Not. Royal Ast. Soc. 348 (Mar., 2004) 811-830, [astro-ph/].

[36] J. E. Taylor and A. Babul, The evolution of substructure in galaxy, group and cluster haloes II. Global properties, Monthly Not. Royal Ast. Soc. 364 (Dec., 2005) 515-534, [astro-ph/].

[37] J. E. Taylor and A. Babul, The evolution of substructure in galaxy, group and cluster haloes III. Comparison with simulations, Monthly Not. Royal Ast. Soc. 364 (Dec., 2005) 535-551, [astro-ph/].

[38] C. Giocoli, G. Tormen, R. K. Sheth, and F. C. van den Bosch, The substructure hierarchy in dark matter haloes, Monthly Not. Royal Ast. Soc. 404 (May, 2010) 502-517, [arXiv: 0911.0436].

[39] S. M. Koushiappas, A. R. Zentner, and A. V. Kravtsov, Distribution of annihilation luminosities in dark matter substructure, Phys. Rev. D 82 (Oct., 2010) 083504, [arXiv: 1006.2391].

[40] E. Komatsu, K. M. Smith, J. Dunkley, C. L. Bennett, B. Gold, G. Hinshaw, N. Jarosik, D. Larson, M. R. Nolta, L. Page, D. N. Spergel, M. Halpern, R. S. Hill, A. Kogut, M. Limon, S. S. Meyer, N. Odegard, G. S. Tucker, J. L. Weiland, E. Wollack, and E. L. Wright, Seven-year Wilkinson Microwave Anisotropy Probe (WMAP) Observations: Cosmological Interpretation, Astrophys. J. Supp. 192 (Feb., 2011) 18, [arXiv:1001.4538].

[41] G. L. Bryan and M. L. Norman, Statistical Properties of X-Ray Clusters: Analytic and Numerical Comparisons, Astrophys. J. 495 (Mar., 1998) 80-99, [astro-ph/].

[42] R. P. van der Marel, M. Fardal, G. Besla, R. L. Beaton, S. T. Sohn, J. Anderson, T. Brown, and P. Guhathakurta, The M31 Velocity Vector. II. Radial Orbit toward the Milky Way and Implied Local Group Mass, Astrophys. J. 753 (July, 2012) 8, [arXiv:1205.6864].

[43] J. Diemand, M. Kuhlen, P. Madau, M. Zemp, B. Moore, D. Potter, and J. Stadel, Clumps and streams in the local dark matter distribution, Nature 454 (Aug., 2008) 735-738, [arXiv: 0805.1244].

[44] J. Wolf, G. D. Martinez, J. S. Bullock, M. Kaplinghat, M. Geha, R. R. Muñoz, J. D. Simon, and F. F. Avedo, Accurate masses for dispersion-supported galaxies, Monthly Not. Royal Ast. Soc. 406 (Aug., 2010) 1220-1237, [arXiv:0908.2995].

[45] J. F. Navarro, C. S. Frenk, and S. D. M. White, The Structure of Cold Dark Matter Halos, Astrophys. J. 462 (May, 1996) 563, [astro-ph/].

[46] J. D. Simon and M. Geha, The Kinematics of the Ultra-faint Milky Way Satellites: Solving the Missing Satellite Problem, Astrophys. J. 670 (Nov., 2007) 313-331, [arXiv:0706.0516].

[47] M. Niederste-Ostholt, V. Belokurov, N. W. Evans, and J. Peñarrubia, Re-Assembling the Sagittarius Dwarf Galaxy, Astrophys. J. 712 (Mar., 2010) 516-526, [arXiv:1002.0266]. 
[48] P. S. Behroozi, C. Conroy, and R. H. Wechsler, A Comprehensive Analysis of Uncertainties Affecting the Stellar Mass-Halo Mass Relation for 0 i z i 4, Astrophys. J. 717 (July, 2010) 379-403, [arXiv: 1001.0015].

[49] A. Klypin, A. V. Kravtsov, O. Valenzuela, and F. Prada, Where Are the Missing Galactic Satellites?, Astrophys. J. 522 (Sept., 1999) 82-92, [astro-ph/].

[50] B. Moore, S. Ghigna, F. Governato, G. Lake, T. Quinn, J. Stadel, and P. Tozzi, Dark Matter Substructure within Galactic Halos, Astrophys. J. Lett. 524 (Oct., 1999) L19-L22, [astro-ph/].

[51] K. R. Stewart, J. S. Bullock, R. H. Wechsler, A. H. Maller, and A. R. Zentner, Merger Histories of Galaxy Halos and Implications for Disk Survival, Astrophys. J. 683 (Aug., 2008) 597-610, [arXiv: 0711.5027].

[52] J. S. Bullock, T. S. Kolatt, Y. Sigad, R. S. Somerville, A. V. Kravtsov, A. A. Klypin, J. R. Primack, and A. Dekel, Profiles of dark haloes: evolution, scatter and environment, Monthly Not. Royal Ast. Soc. 321 (Mar., 2001) 559-575, [astro-ph/].

[53] A. F. Neto, L. Gao, P. Bett, S. Cole, J. F. Navarro, C. S. Frenk, S. D. M. White, V. Springel, and A. Jenkins, The statistics of $\Lambda$ CDM halo concentrations, Monthly Not. Royal Ast. Soc. 381 (Nov., 2007) 1450-1462, [arXiv:0706.2919].

[54] A. V. Macciò, A. A. Dutton, F. C. van den Bosch, B. Moore, D. Potter, and J. Stadel, Concentration, spin and shape of dark matter haloes: scatter and the dependence on mass and environment, Monthly Not. Royal Ast. Soc. 378 (June, 2007) 55-71, [astro-ph/].

[55] A. Cibinel, C. M. Carollo, S. J. Lilly, F. Miniati, J. D. Silverman, J. H. van Gorkom, E. Cameron, A. Finoguenov, P. Norberg, A. Pipino, C. S. Rudick, T. Lu, and Y. Peng, The Zurich Environmental Study (ZENS) of Galaxies in Groups along the Cosmic Web. II. Galaxy Structural Measurements and the Concentration of Morphologically-Classified Satellites in Diverse Environments, ArXiv e-prints (June, 2012) [arXiv:1206.6108]. 